\title{
Quarkonium production and polarization in high-energy collisions
}

\author{
Vincent Cheung ${ }^{\dagger a, b, *}$ and Ramona Vogt $\mathbf{t}^{\dagger a, b}$ \\ ${ }^{a}$ Department of Physics and Astronomy, University of California, Davis, Davis, California 95616, USA \\ ${ }^{b}$ Nuclear and Chemical Sciences Division, Lawrence Livermore National Laboratory, Livermore \\ California, 94551, USA \\ E-mail: hscheung@ucdavis.edu, rlvogt@lbl.gov
}

One of the best ways to understand hadronization in QCD is to study the production of quarkonium. However, the production mechanism of quarkonium is still uncertain. Spin-related measurements like the polarization are strong tests of production models. We show recent results of direct $J / \psi$ production and polarization using the improved color evaporation model in $p+p$ collisions at $\sqrt{s}=7 \mathrm{TeV}$, and compare them with LHC data.

\footnotetext{
*** 10th International Workshop on Charm Physics (CHARM2020), ***

***31 May - 4 June, $2021 * * *$

*** Mexico City, Mexico - Online ***
}

\footnotetext{
* Speaker

${ }^{\dagger}$ This work was performed under the auspices of the U.S. Department of Energy by Lawrence Livermore National Laboratory under Contract DE-AC52-07NA27344 and supported by the U.S. Department of Energy, Office of Science, Office of Nuclear Physics (Nuclear Theory) under contract number DE-SC-0004014.
} 


\section{Introduction}

Since the discovery of the $J / \psi$, several models were developed to describe the production mechanism of heavy quarks, from production in hard processes to the hadronization of the final state. Nonrelativistic QCD (NRQCD) [1] and the color evaporation model (CEM) [2-4] remain the most commonly used models today. However, NRQCD cannot describe $J / \psi$ production and polarization while respecting the universality of the long distance matrix elements (LDMEs) for $p_{T}$ cuts less than twice the mass of the quarkonium state [5, 6]. The LDMEs that are able to describe $J / \psi$ polarization have difficulty describing the $\mathrm{LHCb} \eta_{c}$ production $[7,8]$ using heavy quark spin symmetry [9-11]. On the other hand, while the CEM and the later improved CEM (ICEM) [12] can describe the production distributions, the polarization has not been thoroughly studied. Thus, it is worth revisiting polarization in the (I)CEM, and recent calculations [13, 14] have considered the polarization in hadroproduction.

In this brief overview, we present both the yield and the polarization parameters of direct $J / \psi$ production as a function of $p_{T}$ in the ICEM [12] using the collinear factorization approach. We give a brief introduction of the polarization parameters and the how polarized yields are calculated in the ICEM. We then compare the unpolarized $p_{T}$ distributions and polarization parameters to data. Details of the calculation and comparison to high $p_{T}$ data are covered in a recent paper [15].

\section{Polarization}

The tendency of a quarkonium state to be in a certain angular momentum projection state is known as the polarization. For example, unpolarized $J=1$ quarkonium production means an equal amount of $J_{z}=-1,0,+1$ is produced. Experimentally, the polarization of any $S$-state vector meson is obtained from its decay to $\ell^{+} \ell^{-}$pairs by measuring the distribution of the angle between a chosen $z$-axis and the direction of the positively-charged lepton travels in the quarkonium rest frame. The angular distribution of the production can be expanded in terms of the polarization parameters $\left(\lambda_{\vartheta}\right.$, $\lambda_{\varphi}$, and $\lambda_{\vartheta \varphi}$ ) given by [16],

$$
\frac{d \sigma}{d \Omega} \propto \frac{1}{3+\lambda_{\vartheta}}\left[1+\lambda_{\vartheta} \cos ^{2} \vartheta+\lambda_{\varphi} \sin ^{2} \vartheta \cos (2 \varphi)+\lambda_{\vartheta \varphi} \sin (2 \vartheta) \cos \varphi\right]
$$

where $\vartheta$ and $\varphi$ denote the polar and azimuthal angles respectively, $\lambda_{\vartheta}$ describes the polar anisotropy, $\lambda_{\varphi}$ describes the azimuthal anisotropy, and $\lambda_{\vartheta \varphi}$ describes the polar azimuthal correlation.

Measuring and predicting the polarization is important to understand the acceptance of the $J / \psi$ in the detector. This is because the kinematic acceptance of the detector for measuring quarkonium production depends on the quarkonium polarization hypothesis [17]. Thus, understanding polarization helps reduced systematic uncertainties

\section{Polarized Production in the ICEM}

The ICEM assumes the $J / \psi$ production cross section is a constant fraction of the open $c \bar{c}$ cross section with invariant mass above the mass of the $J / \psi$ but below the hadron threshold, the $D \bar{D}$ pair 
mass. A distinction is also made between the $c \bar{c}$ momentum and the $J / \psi$ momentum in the ICEM. The unpolarized direct $J / \psi$ production cross section in $p+p$ collision in the ICEM is given by [12]

$$
\sigma=\left.F_{J / \psi} \sum_{i, j} \int_{M_{J / \psi}}^{2 m_{D}} d M d x_{i} d x_{j} f_{i}\left(x_{i}, \mu_{F}\right) f_{j}\left(x_{j}, \mu_{F}\right) \hat{\sigma}_{i j \rightarrow c \bar{c}+k}\left(p_{c \bar{c}}, \mu_{R}\right)\right|_{p_{c \bar{c}}=\frac{M}{M_{J} / \psi} p_{\psi}},
$$

where $i$ and $j$ are $q, \bar{q}$ and $g$ such that $i j=q \bar{q}, q g, \bar{q} g$ or $g g, F_{J / \psi}$ is a universal factor at fixed order for direct $J / \psi$ production in the ICEM independent of hadronic projectile and energy, $x$ is the momentum fraction of the parton, and $f\left(x, \mu_{F}\right)$ is the parton distribution function (PDF) for a parton in the proton as a function of $x$ and the factorization scale $\mu_{F}$. Finally, $\hat{\sigma}_{i j \rightarrow c \bar{c}+k}$ are the parton-level cross sections for initial states $i j$ to produce a $c \bar{c}$ pair with a light final state parton $k$. The invariant mass of the $c \bar{c}$ pair, $M$, is integrated from the physical mass of $J / \psi$ $\left(M_{J / \psi}=3.10 \mathrm{GeV}\right)$ to two times the mass of the $D^{0}$ hadron $\left(2 m_{D^{0}}=3.72 \mathrm{GeV}\right)$. Because the $O\left(\alpha_{s}^{3}\right)$ contribution diverges when the light parton is soft, in order to describe the $p_{T}$ distribution at low $J / \psi p_{T}$, the initial state partons are each given a small transverse momentum, $k_{T}$, kick of $\left\langle k_{T}^{2}\right\rangle=1+(1 / 12) \ln (\sqrt{s} / 20 \mathrm{GeV})=1.49 \mathrm{GeV}^{2}$ for $\sqrt{s}=7 \mathrm{TeV}$. The collinear parton distribution functions are then multiplied by the gaussian function $g\left(k_{T}\right)$

$$
g\left(k_{T}\right)=\frac{1}{\pi\left\langle k_{T}^{2}\right\rangle} \exp \left(k_{T}^{2} /\left\langle k_{T}^{2}\right\rangle\right)
$$

assuming the $x$ and $k_{T}$ dependences completely factorize. The same gaussian smearing is applied in Refs. [12, 18, 19].

We consider diagrams at $O\left(\alpha_{s}^{3}\right)$ with the projection operators, $\notin_{\psi}^{*}\left(J_{z}\right)\left(\not p_{\psi}+m_{\psi}\right) /\left(2 m_{\psi}\right)$, applied to the $c \bar{c}[20,21]$ to calculate the partonic cross sections. We include 16 diagrams from the $g g \rightarrow c \bar{c} g$ process, $5 g q \rightarrow c \bar{c} q$ diagrams, $5 g \bar{q} \rightarrow c \bar{c} \bar{q}$ diagrams, and $5 q \bar{q} \rightarrow c \bar{c} g$ diagrams. We assume that the angular momentum of the $c \bar{c}$ (proto- $J / \psi$ ) is unchanged by the transition from the parton level to the hadron level. We then convolute the partonic cross sections with the CT14 PDFs [22]. We restrict the partonic cross section calculations within the perturbative domain by introducing a regularization parameter such that all propagators are at a minimum distance of $Q_{\text {reg }}^{2}=M^{2}$ from their poles, as employed in Ref. [20]. We take the factorization and renormalizaton scales to be $\mu_{F} / m_{T}=2.1_{-0.85}^{+2.55}$ and $\mu_{F} / m_{T}=1.6_{-0.12}^{+0.11}$ respectively, where $m_{T}$ is the transverse mass of the produced charm quark $\left(m_{T}=\sqrt{m_{c}^{2}+p_{T}^{2}}\right.$, where $\left.p_{T}^{2}=0.5 \sqrt{p_{T c}^{2}+p_{T \bar{c}}^{2}}\right)$. We also vary the charm quark mass around $1.27 \pm 0.09 \mathrm{GeV}$. These variations were determined in Ref. [18] where the uncertainties on the total charm cross section were considered.

We consider the polarized production of quarkonium by restricting the final state quarkantiquark pair to be in the desired spin state. The polarization parameters are then calculated in terms of the spin matrix elements $\sigma_{i_{z}, j_{z}}$. In these matrix elements, the quarkonium is assumed to have $J_{z}=i_{z}$ when calculating the scattering matrix element, $\mathcal{M}$. The quarkonium is assumed to take $J_{z}=j_{z}$ in calculating the conjugate, $\mathcal{M}^{*}$. The polarization parameters are calculated using the matrix elements. The polar anisotropy $\left(\lambda_{\vartheta}\right)$, the azimuthal anisotropy $\left(\lambda_{\varphi}\right)$, and polar-azimuthal 
correlation $\left(\lambda_{\vartheta \varphi}\right)$ are given by [16],

$$
\begin{aligned}
\lambda_{\vartheta} & =\frac{\sigma_{+1,+1}-\sigma_{0,0}}{\sigma_{+1,+1}+\sigma_{0,0}}, \\
\lambda_{\varphi} & =\frac{\operatorname{Re}\left[\sigma_{+1,-1}\right]}{\sigma_{+1,+1}+\sigma_{0,0}}, \\
\lambda_{\vartheta \varphi} & =\frac{\operatorname{Re}\left[\sigma_{+1,0}-\sigma_{-1,0}\right]}{\sqrt{2}\left(\sigma_{+1,+1}+\sigma_{0,0}\right)} .
\end{aligned}
$$

Most experiments that report the polarization parameters are consistent with the no polarization hypothesis. However, for a single elementary process, there is no combination that would give $\lambda_{\vartheta}=\lambda_{\varphi}=\lambda_{\vartheta \varphi}=0$. By including polarized production from more than one sub-processes, it is still possible for theory calculations to give an unpolarized production prediction.

The polarization parameters depend on the frame (helicity or Collins-Soper) in which they are calculated and measured. Since the angular distribution itself is rotationally invariant, there are ways to construct invariant polarization parameters from Eqs. (4)-(6). One of the combinations to form a frame-invariant polarization parameter $(\tilde{\lambda})$ is [16]

$$
\tilde{\lambda}=\frac{\lambda_{\vartheta}+3 \lambda_{\varphi}}{1-\lambda_{\varphi}} \text {. }
$$

The choice of $\tilde{\lambda}$ is the same as the polar anisotropy parameter $\left(\lambda_{\vartheta}\right)$ in a frame where the distribution is azimuthally isotropic $\left(\lambda_{\varphi}=0\right)$. We can remove the frame-induced kinematic dependencies when comparing theoretical predictions to data by also considering the frame-invariant polarization parameter, $\tilde{\lambda}$.

\section{Results}

Figure 1 shows the comparison between the ICEM inclusive $J / \psi p_{T}$ distributions at $\sqrt{s}=7 \mathrm{TeV}$ in the forward rapidity region and that measured by LHCb in the forward rapidity region $2<y<4.5$ [24]. Since the detector acceptance depends on the polarization assumption, LHCb presented the data under two assumptions: $J / \psi$ production is either completely longitudinal or completely transverse. Both are shown in Fig. 1. We also compare this calculation with the polarized ICEM in the $k_{T}$-factorization approach [13] and the unpolarized ICEM in the collinear factorization approach [12]. The former also selects $c \bar{c}$ with the same spin as the $J / \psi$ but the latter is a spin-averaged calculation. The uncertainty band of the unpolarized collinear ICEM is constructed in the same way as this calculation. The uncertainty band of the $k_{T}$-factorized ICEM is constructed by varying the renormalization scale in the interval $0.5<\mu_{R} / m_{T}<2$ and varying the charm mass in the interval $1.2<m_{c}<1.5 \mathrm{GeV}$. We find the latest polarized collinear ICEM $p_{T}$ distribution agrees with the data and other calculations in the ICEM in general.

We show the $p_{T}$ dependence of the frame-dependent polarization parameters $\lambda_{\vartheta}, \lambda_{\varphi}$, and $\lambda_{\vartheta \varphi}$ at $\sqrt{s}=7 \mathrm{TeV}$ in the helicity frame and in the Collins-Soper frame in Figs. 2 and 3. We compare the polarization parameters at low and moderate $p_{T}\left(2<p_{T}<15 \mathrm{GeV}\right)$ with the data measured by the LHCb Collaboration [25] and the ALICE Collaboration [? ], where the data are collected in rapidity ranges $2<y<4.5$ and $2.5<y<4$ respectively. A comparison at high $p_{T}\left(14<p_{T}<70 \mathrm{GeV}\right)$ with the data measured by the CMS Collaboration [27] can be found in Ref. [15]. 


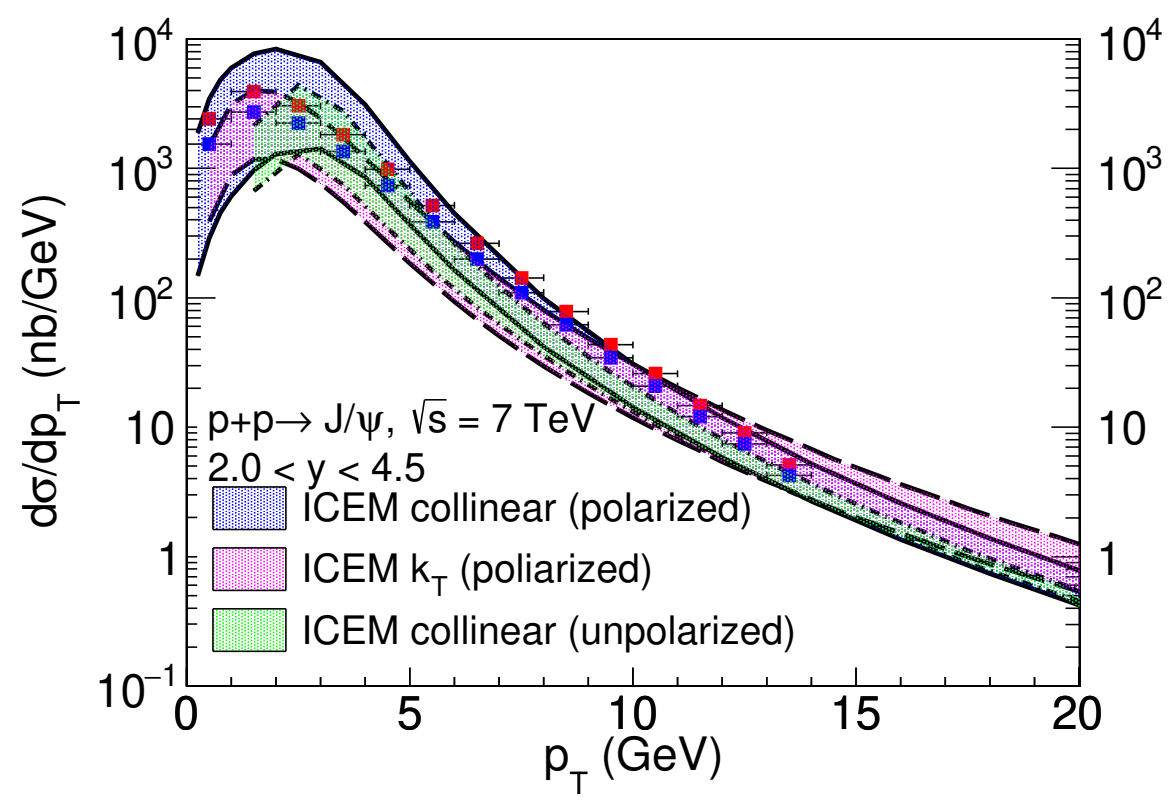

Figure 1: The $p_{T}$ dependence of inclusive $J / \psi$ production at $\sqrt{s}=7 \mathrm{TeV}$ in the polarized collinear ICEM (this calculation) (blue band), in the polarized ICEM using the $k_{T}$-factorization [13] (magenta band), in the unpolarized collinear ICEM [12] (green band). They are compared to the LHCb data [24] assuming that the $J / \psi$ polarization is totally transverse, $\lambda_{\vartheta}=+1$ (red squares), and totally longitudinal, $\lambda_{\vartheta}=-1$ (blue squares). The LHCb data assuming $\lambda_{\vartheta}=0$ lie between the red and blue points and are not shown.

The polar anisotropy parameter $\left(\lambda_{\vartheta}\right)$ reflects the proportion of the $J / \psi$ in each spin projection state, with $\lambda_{\vartheta}=1$ referring to completely transverse production of $J_{z}= \pm 1, \lambda_{\vartheta}=-1$ referring to completely longitudinal production of $J_{z}=0$. At low $p_{T}, \lambda_{\vartheta}$ is close to zero in both the helicity frame and the Collins-Soper frame, indicating equal $J / \psi$ yields in each spin projection state $\left(J_{z}=0, \pm 1\right)$. However, as $p_{T}$ grows larger, the difference between the $\lambda_{\vartheta}$ calculated in the two frames increases with $p_{T}$. We find the transverse component $\left(J_{z}= \pm 1\right)$ falls off more slowly than the longitudinal component $\left(J_{z}=0\right)$ in the helicity frame. As a result, $\lambda_{\vartheta}$ becomes positive as $p_{T}$ increases. This is consistent with the CGC+NRQCD approach at low and moderate $p_{T}$ [29] and a NRQCD calculation at high $p_{T}$ [28]. We find the longitudinal component dominates with increasing $p_{T}$ in the Collins-Soper frame. Thus, $\lambda_{\vartheta}$ becomes negative as $p_{T}$ increases. This relative behavior of $\lambda_{\vartheta}$ in the two frames is expected because the polarization $z$-axes are parallel at $p_{T}=0$ and become orthogonal in the limit $p_{T} \rightarrow \infty$.

The azimuthal anisotropy parameter $\left(\lambda_{\varphi}\right)$ reflects the azimuthal symmetry of $J / \psi$ production. When $\lambda_{\varphi}=0$, the production is azimuthally symmetric. When $\lambda_{\varphi}= \pm 1$, the azimuthal distribution is maximally asymmetric. We note that this parameter strongly depends on the production mechanism as well as the frame the distribution is measured in. In the Collins-Soper frame, this parameter is close to zero over all $p_{T}$ as the matrix element $\sigma_{+1,-1}$ is small relative to $\sigma_{+1,+1}$ and $\sigma_{0,0}$. This means that the $z_{C S}$-axis is approximately the azimuthal symmetry axis. In the helicity frame, the matrix element $\sigma_{+1,-1}$ is negative and becomes more negative. As a result, $\lambda_{\varphi}$ becomes negative as $p_{T}$ grows larger, showing that the $z_{H X}$-axis is not the symmetry axis of the distribution. However, 

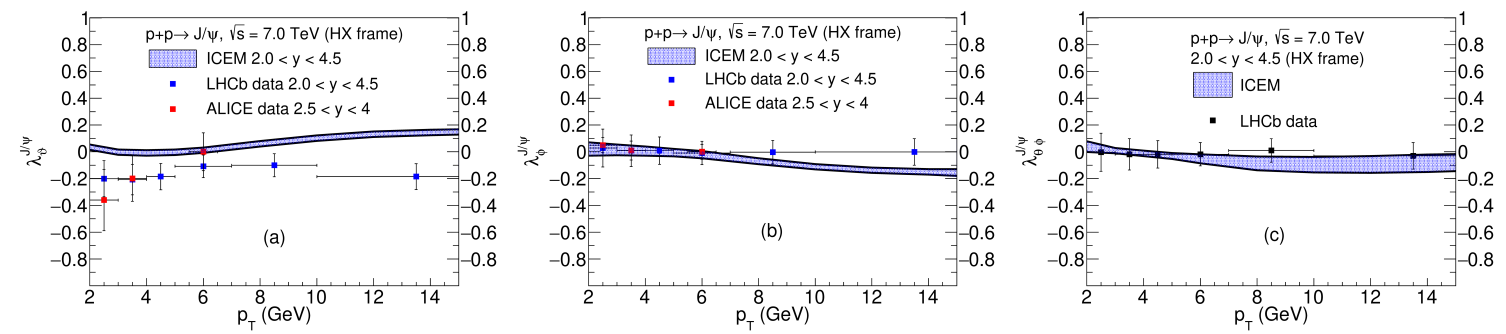

Figure 2: The polar anisotropy parameter $\left(\lambda_{\vartheta}\right)$ (a), the azimuthal anisotropy parameter $\left(\lambda_{\varphi}\right)(\mathrm{b})$, and the polar-azimuthal correlation parameter $\left(\lambda_{\vartheta \varphi}\right)$ (c) in the helicity frame at $\sqrt{s}=7 \mathrm{TeV}$ in the ICEM. The combined mass, renormalization scale, and factorization scale uncertainties are shown in the band and compared to the LHCb data [25] (blue) and the ALICE data [? ] (red).
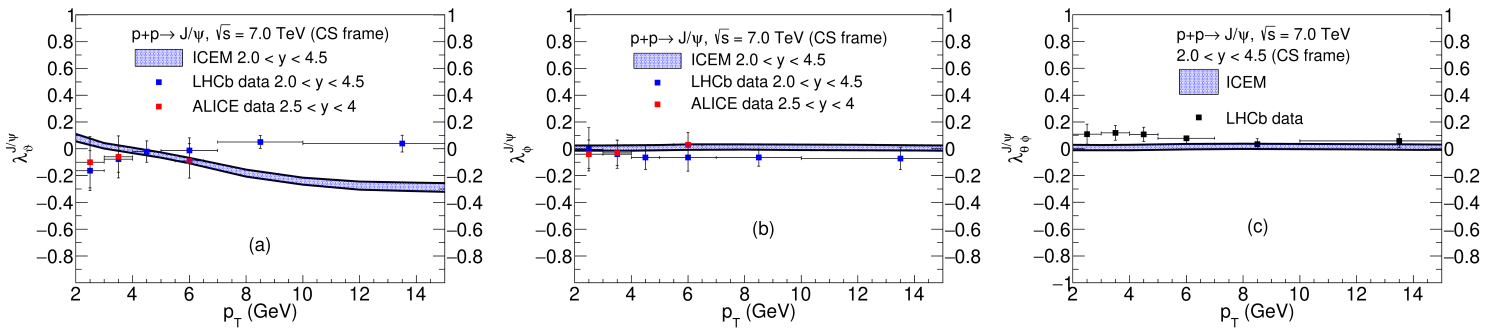

Figure 3: The polar anisotropy parameter $\left(\lambda_{\vartheta}\right)$ (a), the azimuthal anisotropy parameter $\left(\lambda_{\varphi}\right)$ (b), and the polar-azimuthal correlation parameter $\left(\lambda_{\vartheta}\right)$ (c) in the Collins-Soper frame at $\sqrt{s}=7 \mathrm{TeV}$ in the ICEM. The combined mass, renormalization scale, and factorization scale uncertainties are shown in the band and compared to the LHCb data [25] (blue) and the ALICE data [? ] (red).

the distribution itself is rotationally invariant. The discrepancy between $\lambda_{\varphi}$ in these two frames is a combination of two factors: $z_{C S}$ and $z_{H X}$ becomes approximately orthogonal as $p_{T}$ increases and production is not spherically symmetric.

The polar-azimuthal correlation parameter $\left(\lambda_{\vartheta \varphi}\right)$ describes the angular correlation between $2 \vartheta$ and $\varphi$. When $\lambda_{\vartheta \varphi}=0$, the two angles are uncorrelated and as $\lambda_{\vartheta \varphi}$ departs from 0 , the behavior of the distribution becomes similar at locations where $2 \vartheta=\varphi$. In both the helicity frame and the Collins-Soper frame, $\lambda_{\vartheta \varphi}$ is consistent with 0 , which agrees with the data.

We observe the ICEM frame-dependent polarization parameters is in better agreement with the measured data in the helicity frame than in the Collins-Soper frame at high $p_{T}$. However, even though we are switching from one frame to another, we are still comparing the same angular distributions. In order to remove frame-induced kinematic dependencies, we compute the frameinvariant polarization parameter $\tilde{\lambda}$ as a function of $p_{T}$ using $\lambda_{\vartheta}$ and $\lambda_{\varphi}$. We compare $\tilde{\lambda}$ as a function of $p_{T}$ with the data from LHCb in the helicity and the Collins-Soper frames in Fig. 4. Since the azimuthal anisotropy parameter $\lambda_{\varphi}$ in the Collins-Soper frame is close to zero in all $p_{T}$ ranges considered, the $p_{T}$ dependence of the invariant polarization parameter $\tilde{\lambda}$ is very similar to that of $\lambda_{\vartheta}$ in the Collins-Soper frame. We find the curves are generally within $1 \sigma$ of the low and moderate $p_{T}$ data. Our direct $J / \psi$ invariant polarization results are in reasonable agreement with the measured data despite a decrease in $\tilde{\lambda}$ with $p_{T}$. We note that including feed down from higher mass states could change the slope in this $p_{T}$ range. We also find our results are in agreement with 


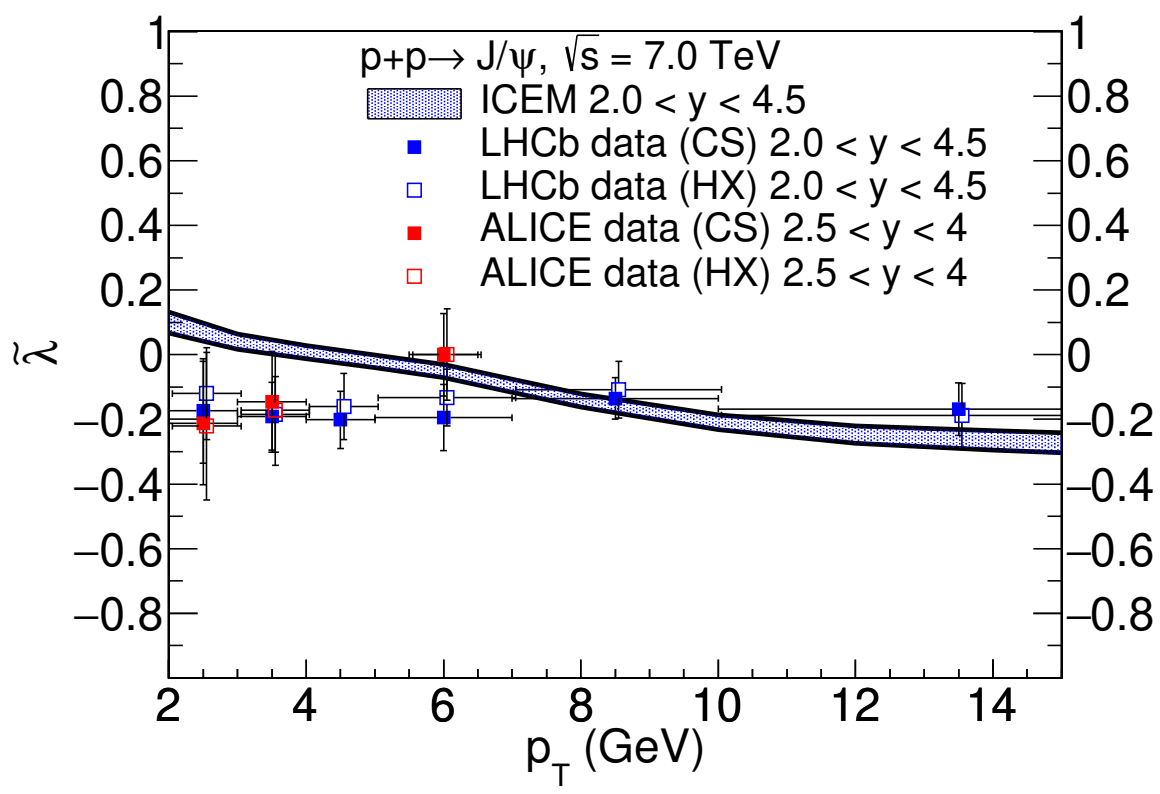

Figure 4: (Color online) The $p_{T}$ dependence of the frame-invariant polarization parameter, $\tilde{\lambda}$, in the ICEM compared to LHCb data [25] (blue) and the ALICE data [26] (red). The data in the helicity frame is displaced by $0.05 \mathrm{GeV}$ for visualization purposes.

the $J / \psi$ invariant polarization found in the CGC+NRQCD approach at low and moderate $p_{T}$ [29].

\section{Conclusion}

We have presented the transverse momentum dependence of the direct $J / \psi$ polarization including the frame-invariant parameter in $p+p$ collisions in the improved color evaporation model in the collinear factorization approach. We also compare the $p_{T}$ distribution to data for inclusive $J / \psi$ production measured by the LHCb. We find the direct $J / \psi$ production is consistent with the unpolarized data at small and moderate $p_{T}$ and becomes longitudinal in the high $p_{T}$ limit. In the future, we anticipate the feed down from $P$ states can explain the discrepancies in high $p_{T}$.

\section{References}

[1] W. Caswell and G. P. Lepage, Phys. Lett. B 167, 437 (1986).

[2] V. D. Barger, W. Y. Keung, and R. J. N. Phillips, Phys. Lett. 91B, 253 (1980).

[3] V. D. Barger, W. Y. Keung, and R. J. N. Phillips, Z. Phys. C 6, 169 (1980).

[4] R. Gavai, D. Kharzeev, H. Satz, G. A. Schuler, K. Sridhar, and R. Vogt, Int. J. Mod. Phys. A 10, 3043 (1995).

[5] G. T. Bodwin, H. S. Chung, U. R. Kim, and J. Lee, Phys. Rev. Lett. 113, 022001 (2014).

[6] P. Faccioli, V. Knünz, C. Lourenco, J. Seixas, and H. K. Wöhri, Phys. Lett. B 736, 98 (2014). 
[7] H. Han, Y. Q. Ma, C. Meng, H. S. Shao, and K. T. Chao, Phys. Rev. Lett. 114, 092005 (2015).

[8] H. F. Zhang, Z. Sun, W. L. Sang, and R. Li, Phys. Rev. Lett. 114, 092006 (2015).

[9] M. Neubert, Phys. Rep. 245, 259 (1994).

[10] F. De Fazio, in At the Frontier of Particle Physics/Handbook of QCD, edited by M. A. Shifman (Wolrd Scientific, Singapore, 2000) p.1671.

[11] R. Casalbuoni, A. Deandrea, N. Di Bartolomeo, R. Gatto, F. Feruglio, and G. Nardulli, Phys. Rep. 281, 145 (1997).

[12] Y. Q. Ma and R. Vogt, Phys. Rev. D 94, 114029 (2016).

[13] V. Cheung and R. Vogt, Phys. Rev. D 98, 114029 (2018).

[14] V. Cheung and R. Vogt, Phys. Rev. D 99, 034007 (2019).

[15] V. Cheung and R. Vogt, Phys. Rev. D (to be published) [accepted for publication].

[16] P. Faccioli, C. Lourenco, J. Seixas, and H. K. Wohri, Eur. Phys. J. C 69, 657 (2010).

[17] G. Aad et al. (ATLAS Collaboration), Nucl. Phys. B 850, 387-444 (2011).

[18] R. E. Nelson, R. Vogt, and A. D. Frawley, Phys. Rev. C 87, 014908 (2013).

[19] M. L. Mangano, P. Nason and G. Ridolfi, Nucl. Phys. B 373, 295-345 (1992).

[20] S. P. Baranov, Phys. Rev. D 66, 114003 (2002).

[21] E. L. Berger and D. L. Jones, Phys. Rev. D 23, 1521-1530 (1981).

[22] S. Dulat, T. J. Hou, J. Gao, M. Guzzi, J. Huston, P. Nadolsky, J. Pumplin, C. Schmidt, D. Stump and C. P. Yuan, Phys. Rev. D 93, 033006 (2016).

[23] J. C. Collins and D. E. Soper, Phys. Rev. D 16, 2219 (1977).

[24] R. Aaij et al. (LHCb Collaboration), Eur. Phys. J. C 71, 1645 (2011).

[25] R. Aaij et al. (LHCb Collaboration), Eur. Phys. J. C 73, 2631 (2013).

[26] B. Abelev et al. (ALICE Collaboration), Phys. Rev. Lett. 108, 082001 (2012).

[27] S. Chatrchyan et al. (CMS Collaboration), Phys. Lett. B 727, 381-402 (2013).

[28] K. T. Chao, Y. Q. Ma, H. S. Shao, K. Wang and Y. J. Zhang, Phys. Rev. Lett. 108, 242004 (2012).

[29] Y. Q. Ma, T. Stebel and R. Venugopalan, JHEP 12, 057 (2018). 